\title{
Surgical treatment of advanced anterior wall and apical vaginal prolapse using the anchorless self-retaining support implant: long-term follow-up
}

\author{
Gil Levy $^{1}$ (D) Anna Padoa $^{2} \cdot$ Naama Marcus $^{3} \cdot$ Anat Beck $^{4} \cdot$ Zoltan Fekete $^{5} \cdot$ Mauro Cervigni $^{6}$
}

Received: 3 August 2021 / Accepted: 1 November 2021 / Published online: 13 January 2022

(c) The International Urogynecological Association 2021

\begin{abstract}
Introduction and hypothesis Following health notification by the FDA in 2008 of serious complications with transvaginal mesh for anterior pelvic organ prolapse, there has been a return to native tissue repairs. Earlier work with a self-retaining support (SRS) implant showed a high anatomical success rate with minimal implant-related complications over a mediumterm follow-up. It is proposed that post-implant complications are more a consequence of the method of mesh anchoring rather than the implant itself. Our system incorporates an ultralight mesh with a frame that provides level I, II, and III support without the need for fixation. The first long-term outcomes of SRS implantation are presented.

Methods A prospective multicenter trial was conducted using two consecutive identical protocols of the use of the SRS implant in women with symptomatic anterior compartment prolapse extending their follow-up to 36 months. Anatomical success (Pelvic Organ Prolapse Quantification stage 0 or 1 or a $\mathrm{Ba} \leq-2$ ) was recorded along with subjective success as defined by regular quality-of-life (PFDI-20 and Pelvic Organ Prolapse/Urinary Incontinence Sexual Questionnaire) assessments.

Results Sixty-seven patients completed 36 months of follow-up. Mean Ba measurements improved from 3.1 ( -1 to 6) $\mathrm{cm}$ to $-2.8(-1$ to -3$) \mathrm{cm}$ and $\mathrm{C}$ point from $0.4(-8$ to 6$) \mathrm{cm}$ to $-6.9(-10 \mathrm{~cm}$ to 1$) \mathrm{cm}$. accumulating to a significant anatomical success rate of $94.3 \%$. Subjective success based on question \#3 of the PFDI-20, analyzed for the index surgical compartment, reached $95.7 \%$. Post-operative complications included 2 cases of urinary retention, 1 minor frame exposure, 1 case of delayed voiding dysfunction, and 2 cases of de novo stress urinary incontinence. Untreated pre-operative second-degree Bp measurements had increased in $27 \%$ at follow-up.

Conclusion The long-term outcome of the SRS implant shows an excellent subjective and objective success with minimal risk of complications or need for reintervention.
\end{abstract}

Keywords Self-retaining support implant · Vaginal mesh · Pelvic organ prolapse

\section{Introduction}

Gil Levy

2gillevy@gmail.com

Assuta University Hospital, Ashdod, Israel

2 Shamir Medical Center, Zrifin, Israel

Ziv Medical Center, Zfad, Israel

4 Maynei Hayeshua Hospital, Bnei Brak, Israel

5 Szeged University Hospital, Szeged, Hungary

6 "La Sapienza" Univ.-Polo Pontino, ICOT-Latina, Italy
Over the last decade there has been increasing concern about the use of vaginal mesh in patients presenting with symptomatic pelvic organ prolapse (POP). This has prompted a return toward native tissue repairs (NTRs) and sacrocolpopexy in patient management $[1,2]$. The anxieties regarding mesh-related complications such as exposure and extrusion, chronic pelvic pain and disabling dyspareunia [3] prompted the FDA in 2008 to issue a public health notice designed to inform both patients and their physicians of potentially serious side effects with the use of transvaginal mesh [4]. In 2011, with the rash of adverse events reported by practitioners, the FDA reissued a safety notice providing new data on additional problems that included 
mesh contraction and the effects of secondary inflammatory responses such as vaginal shortening and pain (http:// www.fda.gov/medicaldevices/safety/alertsandnotices/ucm 26 2435.htm). A consequence was the reclassification in early 2016 of transvaginal mesh devices from a moderate (class II) to a high (class III) risk and the need with the introduction of any new device for formal FDA premarket approval (PMA; https://www.fda.gov/medical-devices/premarketsubmissions/premarket-approval-pma). As manufacturers were unable in these PMAs to demonstrate that the benefits of their mesh products outweighed the risk in comparison with NTR, the FDA ordered the withdrawal of these mesh products from the market in the United States in April 2019.

Recent studies have reported transvaginal mesh-related adverse events particularly associated with anchoring of the implant to pelvic myofascial structures. In this circumstance, it is believed that it is the anchoring of the mesh rather than the mesh itself and the specific fixation technique that are implicated in the pathogenesis of post-operative complications [5]. Consequently, a new implant, the self-retaining support (SRS) implant (Lyra Medical, Binyamina, Israel) was developed. The SRS implant was designed specifically to retain the benefits of mesh whilst avoiding these problems attributed to soft-tissue anchoring. The SRS implant consists of a flat but stretched ultra-light polypropylene mesh, mimicking the anatomical shape and size of the pubo-cervical fascia, which has been primarily damaged in POP patients [6]. The mesh is kept flattened by a solid frame that extends to the ischial spines and which provides level I, level II, and level III support [7]. Preliminary data obtained by our group in a cadaveric model have shown accurate deployment of the frame medial and proximal to the ischial spines, with the lateral arms following along the line of the arcus tendineus fascia pelvis (ATFP) [8]. Early clinical experience with this anchorless device in 20 women with anterior vaginal wall prolapse (with and without apical prolapse) showed implant safety with normalization of the post-operative Pelvic Organ Prolapse Quantification (POP-Q) measurements [9]. Over a medium-term follow-up of 27.7 months in 70 patients implanted with the SRS device there was an anatomical success rate of $94.3 \%(\mathrm{Ba} \leq-2 \mathrm{~cm})$ without any cases of mesh erosion or chronic pain [10]. In every case the Ba point was above the hymen. We present the longer-term outcomes of surgery with the SRS implant where there has been an emphasis on the rate of anatomical success along with a record of patient-reported post-operative symptoms.

\section{Materials and methods}

This prospective, multicenter international trial assessed the safety and efficacy outcomes of SRS (Lyra Medical, Binyamina, Israel) implantation. The study was approved by the Health Ministries of Israel and Hungary and by each institutional Ethics Committee of the four participating medical centers. The initial research protocol studied 20 patients with a 12-month follow-up, which was then extended to a follow-up of 36 months (ClinicalTrials.gov NCT02209337). Following these initial results, a second protocol was commenced recruiting an additional 50 patients (NCT03195361). The study protocols of these two trials were identical, permitting the use of combined data for the purposes of this current report. The protocol includes those patients with anterior compartment prolapse ( $\geq$ stage 2 ), although the vast majority of patients $(90 \%)$ presented with stages 3 or 4 anterior vaginal wall prolapse (with or without apical prolapse). All patients received a detailed explanation of the risks involved with transvaginal implants and each participant in the analysis signed an informed consent, which had been translated into their local language. Exclusion criteria included asymptomatic prolapse, patients with previous vaginal mesh surgery, patients planning pregnancy and any condition that in the judgment of the investigators would interfere with the subject's ability to provide informed consent and comply with study instructions, place the subject at increased risk, or that might confound interpretation of the study results. Patients undergoing SRS implantation and a concomitant procedure were included for analysis. Occult stress urinary incontinence (SUI) was evaluated with coughing during vaginal examination with manual reduction of the prolapse.

Data pertaining to the prior medical history, along with the pre- and post-operative POP-Q measurements and reported quality-of-life (QoL) scores (the PFDI-20 and Pelvic Organ Prolapse/Urinary Incontinence Sexual Questionnaire [PISQ-12]) were collated using validated versions [11]. The PFDI-20 QoL questionnaire includes three domains; a Pelvic Organ Prolapse Distress Inventory 6 (POPDI-6), a Urinary Distress Inventory (UDI-6), and a Colorectal-Anal Distress Inventory 8 (CRADI-8). Within the questionnaire each answer can have five grades resulting in a point score between 0 and 4 . The mean value of the total number of the items within each domain that is answered is then multiplied by 25 in order to obtain the domain score (range 0 to 100). The total PFDI-20 is recorded out of a maximum of 300 by adding the three domain scores. [12].

Intra-operative information, including operative time, estimated blood loss, complications, etc., and post-operative adverse events were all recorded. Post-operative follow-up was conducted in the clinic at 2 weeks and then subsequently at 2, 6, 12, 24, and 36 months. Each patient was examined at least once by a senior gynecologist other than the surgeon. During each visit patients underwent a vaginal examination and in addition answered the structured pelvic floor symptom questionnaires (PFDI-20 and PISQ-12), which were administered from the 6-month follow-up visits thereafter. 
Subjective outcome assessment was conducted by participant self-completed questionnaires, thus avoiding interviewer bias. All POP-Q measurements were measured by a qualified urogynecologist with the patient positioned in the semi-Fowler position and with measurements taken during a Valsalva maneuver. The anterior and apical compartments' measurements were used for success evaluation and the posterior compartment's measurements for rectocele evaluation. The need for repeat operations and any type of intervention for the relief of pelvic floor dysfunction (including the use of pessaries and/or pelvic floor rehabilitation) were all recorded. There were several specific parameters of success. Patients were considered as anatomical treatment successes if at their last follow-up the measured POP-Q stage was 0 or $1(\mathrm{Ba} \leq-2)$. Subjective success was also recorded where the patient's answer to the third question of the PFDI-20 ("Do you usually have a bulge or something falling out that you can see or feel in your vaginal area?") was $<2$ (i.e., not present or not at all). A further measure of success was if there was no re-treatment for prolapse of the index segment up until the time of closure of the study.

\section{Surgical technique}

The procedure has been previously described [9]. Briefly, an initial anterior colpotomy is performed and dissection is continued laterally as far as the ischial spine on both sides. The SRS implant is composed of an ultra-light, titanized polypropylene surgical mesh $\left(16 \mathrm{~g} / \mathrm{m}^{2}\right)$ that is stretched and retained in place by a U-shaped flexible frame. The device is inserted between the bladder and the vaginal mucosa with the lateral arms following the anatomy of the ATFP. The frame of the implant is positioned symmetrically without tension, with the mesh stretched under the bladder and the connecting bridge deployed under the pubic symphysis. Depending on whether the patient was undergoing a concomitant hysterectomy, either the cervix or the apex of the vaginal vault was then sutured onto the free (proximal) edge of the mesh. Apart from this suture, no other anchoring is used. Hysterectomy could be added to the planned surgery in those cases where ultrasound revealed a uterine abnormality.

\section{Statistical analysis}

The first protocol was "first in human" and did not use a formal statistical calculation. In the following protocol, for safety analysis purposes, a sample size of 70 subjects used a pooled analysis method consisting of 20 subjects from the initial protocol and 50 subjects from the second protocol.

Data were analyzed using SPSS version 26.0 (Chicago, IL, USA) software. The ANOVA test was used assuming the null hypothesis that the means of all subgroups were equal. The changes in measured points ( $\mathrm{Aa}, \mathrm{Ba}$, and $\mathrm{C}$ ) were assessed by two-way ANOVA for all subjects at each visit comparing these with the final visit. The pre- and post-operative POP-Q measurements were compared using the Wilcoxon rank test. Missing items in the PFDI-20 were ignored using the mean from answered items only. Comparisons were made where the minimally clinically important difference (MCID) per domain was 15 points (total 45 points). $p$ values $<0.05$ were considered significant.

\section{Results}

There were four participating medical centers in this study (Shamir Medical Center, Zrifin, Israel; Ziv Medical Center, Zafed, Israel; Mayanei HaYeshua, Bnei Brak, Israel and Szeged University Hospital, Szeged, Hungary). In these centers, six participating urogynecologists performed all the procedures, with each operating consultant formally trained and accredited in pelvic floor reconstructive surgery. There were a total of 70 women (mean age 63 years; range 43-79 years) recruited over two discrete study periods (one commencing in September 2014 and the other running between March 2016 and December 2020). Figure 1 shows a flowchart of the patient cohort, with 1 patient who declined longer-term follow-up after her 12-month visit and a further 2 cases from the second study period who were lost to follow-up. There were 10 patients who did not attend their last office visit because of the COVID-19 pandemic but who underwent a telephone interview only. Overall, there were 67 patients in the analysis who completed 36 months of follow-up. The median follow-up of the available cohort was 38.4 months (range 12.5-51.3 months).

The basic patient demographic data are shown in Table 1. The mean parity was 4.6 (range 1-16) with a mean BMI of $26.3 \mathrm{~kg} / \mathrm{m}^{2}$ (range 20.3-26.6). Fifteen (21\%) were diabetic, with 6 patients $(8.6 \%)$ having undergone a prior hysterectomy and $6(8.6 \%)$ a previous POP repair. An SRS implant procedure was performed along with a hysterectomy in 10 (14\%), a mid-urethral sling (MUS) in 14 (20\%) and a posterior colporrhaphy in $15(21 \%)$ of the patients. There was no difference in the surgical outcome of the anterior and apical repair when comparing the cases with or without hysterectomy or MUS. Telephone interviews included the routine follow-up questions along with administration of the PFDI-20 and the PISQ-12 questionnaires. Of the 67 patients 57 underwent a vaginal examination and a QoL questionnaire at 36 months of follow-up. Ten patients completed their 36-month QoL questionnaire but with variable clinical examination (4 with only a 1-year vaginal examination and 6 with an examination at 2 years).

Table 2 shows the mean POP-Q measurements and POP stage before surgery and at the last post-operative visit. The mean pre-operative $\mathrm{Aa}, \mathrm{Ba}$, and $\mathrm{C}$ values were $2 \mathrm{~cm}(-1$ 
Enrollment ( $n=70)$, no patient was excluded during screening, all patients received treatment with the SRS Implant

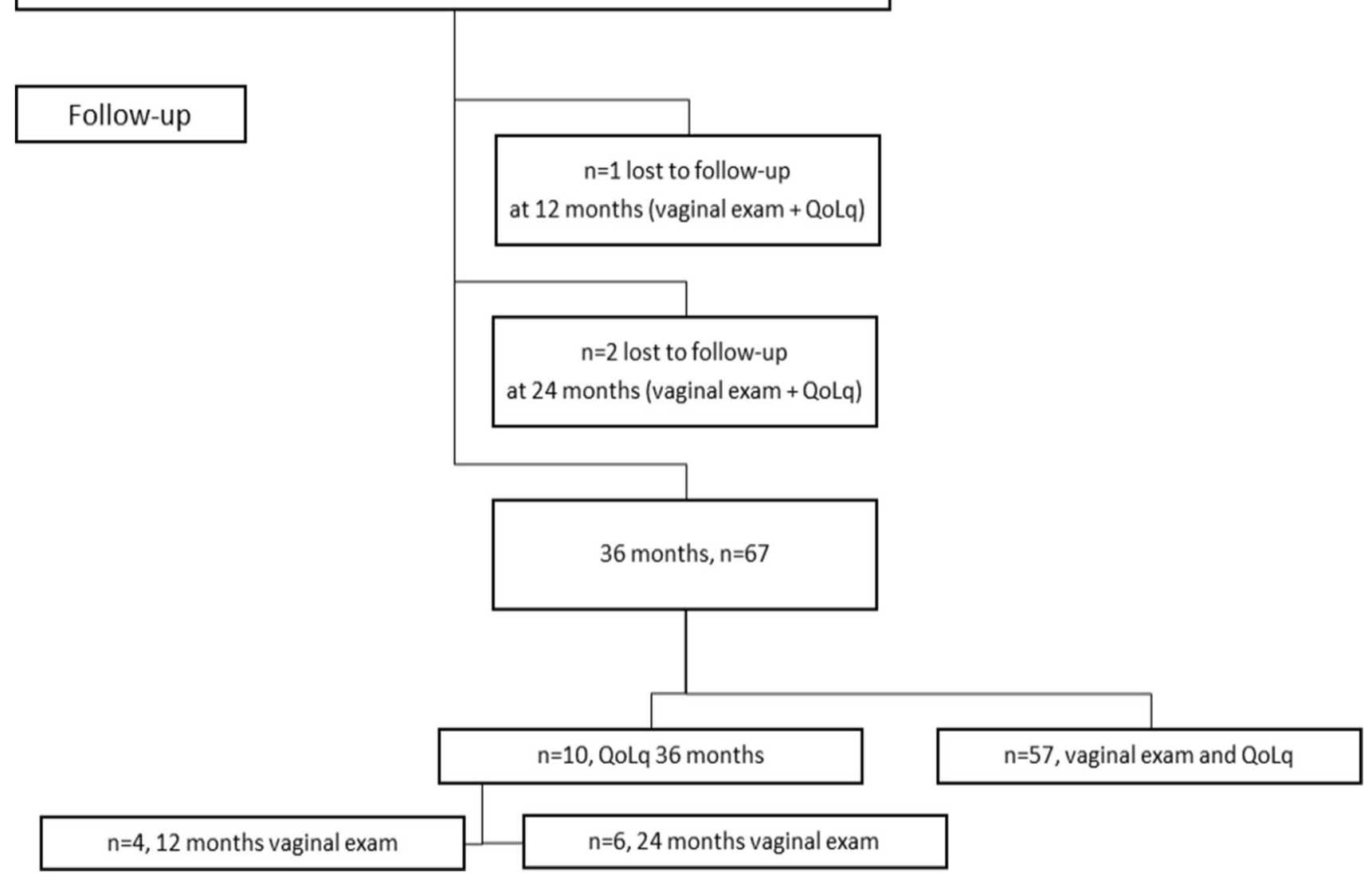

Fig. 1 Flow chart of the patient cohort undergoing SRS implantation and follow-up. QoLq quality of life questionnaire

Table 1 Patient demographic data $(N=70$; median follow-up 38.4 months. (range 12.5-51.3)

\begin{tabular}{lll}
\hline Parameter & Average or $n$ & $\%$ \\
\hline Mean age in years (range) & $63(43-79)$ & \\
Mean parity (range) & $4.6(1-16)$ & \\
Mean BMI, kg $/ \mathrm{m}^{2}$ (range) & $26.3(20.3-36.6)$ & \\
Hypertension & 28 & 40 \\
Diabetes & 15 & 21 \\
Smoker & 10 & 14 \\
Previous hysterectomy & 6 & 8.6 \\
Previous POP surgery & 6 & 8.6 \\
Concomitant hysterectomy & 10 & 14 \\
Concomitant MUS & 14 & 20 \\
Concomitant posterior colporrhaphy & 15 & 21 \\
\hline
\end{tabular}

Average is mean

$B M I$ body mass index, $P O P$ pelvic organ prolapse, $M U S$ mid-urethral sling

to $3 \mathrm{~cm}), 3.1 \mathrm{~cm}(-1$ to $6 \mathrm{~cm})$, and $0.4 \mathrm{~cm}(-8$ to $6 \mathrm{~cm})$ respectively. Before surgery, 7 of the patients $(10 \%)$ were stage $2,51(73 \%)$ stage 3 , and $12(17 \%)$ stage 4 . In the cohort
Table 2 Pelvic Organ Prolapse Quantification measurements before surgery and at last post-operative visit

\begin{tabular}{|c|c|c|}
\hline Variable & $\begin{array}{l}\text { Baseline (pre-oper- } \\
\text { ative) }\end{array}$ & $\begin{array}{l}\text { Last post-operative } \\
\text { VE }\end{array}$ \\
\hline \multicolumn{3}{|c|}{ Anterior compartment } \\
\hline Mean Aa (range) & $2.0(-1$ to 3$)$ & $-2.9(-3 \text { to }-1)^{*}$ \\
\hline Mean $\mathrm{Ba}$ (range) & $3.1(-1$ to 6$)$ & $-2.8(-3 \text { to }-1)^{*}$ \\
\hline \multicolumn{3}{|c|}{ Apical compartment } \\
\hline Mean C (range) & $0.4(-8$ to 6$)$ & $-6.9(-10 \text { to } 1)^{*}$ \\
\hline Stage $0(\%)$ & 0 & $60(85.7)$ \\
\hline Stage $1(\%)$ & 0 & $6(8.6)$ \\
\hline Stage $2(\%)$ & $7(10)$ & $4(5.7)$ \\
\hline Stage $3(\%)$ & $51(73)$ & $0(0)$ \\
\hline Stage $4(\%)$ & $12(17)$ & $0(0)$ \\
\hline
\end{tabular}

Stages of prolapse considered the anterior and apical measurements $V E$ vaginal examination $* p<0.05$

16 patients $(23 \%)$ had anterior vaginal wall prolapse only (i.e., point $\mathrm{C}$ was $<-1 \mathrm{~cm}$ ), whereas the remainder $(77 \%)$ 
presented with both anterior and apical prolapse. The mean operative time for SRS implantation was $24.7 \mathrm{~min}$ (range, 10-50 min) with a mean estimated blood loss of $155 \mathrm{ml}$ (range 25-500 ml). There were no intra-operative complications. One patient required a transfusion (one unit of packed red cells) after surgery and $2(2.8 \%)$ patients had transient urinary retention, both managed with temporary catheterization. One patient (1.8\%) presented 8 months after SRS implantation with vaginal discharge that was secondary to frame erosion. In this case, the larger frame (75-mm lateral arm) had been used and the exposed part of the erosion was excised under local anesthesia with no further complications. It is assumed that an oversized implant accompanied by an incomplete dissection could have resulted in establishment of sufficient pressure by the implant on the vaginal mucosa to result in protrusion of the frame. One additional patient complained of voiding difficulty at the 12-month follow-up visit although residual post-voiding urinary volumes were normal and urethral X-ray examination showed no urethral obstruction. In this case, the sub-urethral bridge part of the frame was thought to be implicated and was removed. By the 2-year visit, her symptoms had not improved and she has been offered urethral dilatation for symptom improvement. A further 2 (2.8\%) patients developed new-onset SUI, both undergoing an MUS procedure following a period of unsuccessful pelvic floor muscle rehabilitation therapy. Nine patients were treated for urinary tract infection (UTI) during the post-operative period, which responded well to oral antibiotics and did not require readmission. According to the Clavien-Dindo classification, 2 patients, who required removal of the SRS bridge, constituted the only grade IIIa complication group. One case of blood transfusion and 9 cases of UTI treated with antibiotics constitute the group II complications. There were no post-operative complications in group IV or V.

The mean post-operative $\mathrm{Aa}, \mathrm{Ba}$, and $\mathrm{C}$ values at the final visit were $-2.9 \mathrm{~cm}(-3$ to $-1 \mathrm{~cm}),-2.8 \mathrm{~cm}(-3$ to $-1 \mathrm{~cm})$ and $-6.9 \mathrm{~cm}(-10$ to $1 \mathrm{~cm})$ respectively (Table 2). At the final visit, 60 of the patients $(85.7 \%)$ were stage $0,6(8.6 \%)$ were stage 1 , and $4(5.7 \%)$ were stage 2 above the hymen (Ba $<0$ ) with no stage 3 or stage 4 cases (Table $2 ; p<0.05$ ). One patient presented with the point $\mathrm{C}=1 \mathrm{~cm}$ who was classified as a case of cervical elongation $(D=-6 \mathrm{~cm})$ in accordance with the definition by Nosti et al. [13]. This case was not considered an anatomical failure, resulting in an anatomical success rate of $94.3 \%$ (stage 0 or 1) and with a $100 \%$ anatomical success rate using a current success criterion of a measured $\mathrm{Ba} \leq 0 \mathrm{~cm}$. Table 3 shows the impact of different clinical factors in post-operative measurable POP parameters with no effect on the clinical outcome of BMI, smoking, diabetes, or parity. There were no cases of re-operation secondary to prolapse recurrent in the treated compartment. The subjective success rate was reliant upon the answer to question number 3 of the PFDI-20 questionnaire with any positive response (a score of 2 or greater) recorded as a subjective failure. At the final visit there were 8 patients (11.4\%)

Table 3 Risk factors associated with measurable prolapse surgery outcome

\begin{tabular}{|c|c|c|c|c|c|c|}
\hline & \multicolumn{3}{|l|}{ Pre-operative } & \multicolumn{3}{|l|}{ Post-operative } \\
\hline & \multicolumn{2}{|l|}{ Anterior } & \multirow{2}{*}{$\begin{array}{l}\text { Apical } \\
\text { C }\end{array}$} & \multicolumn{2}{|l|}{ Anterior } & \multirow{2}{*}{$\begin{array}{l}\text { Apical } \\
\text { C }\end{array}$} \\
\hline & $\overline{\mathrm{Aa}}$ & $\mathrm{Ba}$ & & $\mathrm{Aa}$ & $\mathrm{Ba}$ & \\
\hline \multicolumn{7}{|l|}{ BMI $\left(\mathrm{kg} / \mathrm{m}^{2}\right)$} \\
\hline$<25(n=19)$ & $1.8(-1$ to 3$)$ & $2.8(-2$ to 6$)$ & $0.3(-3$ to 5$)$ & $-3(-3)$ & $-2.9(-3$ to -2$)$ & $-7.1(-9$ to -1$)$ \\
\hline$>25(n=33)$ & $2.2(0$ to 3$)$ & $3.5(1.6$ to 0.3$)$ & $0.5(2.5$ to 0.4$)$ & $-2.8(-3$ to -1$)$ & $-2.7(-3$ to -1$)$ & $-7.3(-10$ to -1$)$ \\
\hline$\geq 30(n=15)$ & $1.8(-1$ to 3$)$ & $2.8(-2$ to 6$)$ & $0.1(-8$ to 5$)$ & $-3(-3)$ & $-2.9(-3$ to -2$)$ & $-5.8(-10$ to 0$)$ \\
\hline$p$ value & 0.482 & 0.395 & 0.907 & 0.065 & 0.175 & 0.215 \\
\hline \multicolumn{7}{|l|}{ Smoking } \\
\hline Smoker $(n=10)$ & 1.0 (-1 to 3$)$ & $1.4(-2$ to 4$)$ & $-0.9(-3$ to 3$)$ & $-2.7(-3$ to -1$)$ & $-2.7(-3$ to -1$)$ & $-7.6(-9.5$ to -5$)$ \\
\hline $\begin{array}{l}\text { Nonsmoker } \\
(n=60)\end{array}$ & $2.2(-1$ to 3$)$ & $3.3(-2$ to 6$)$ & 0.5 ( -8 to 6$)$ & $-2.9(-3$ to -1$)$ & $-2.9(-3$ to -1$)$ & $-6.8(-10$ to 1$)$ \\
\hline$p$ value & 0.051 & 0.072 & 0.138 & 0.405 & 0.813 & 0.426 \\
\hline \multicolumn{7}{|l|}{ Diabetes } \\
\hline Diabetic $(n=15)$ & $2.2(-1$ to 3$)$ & 3.8 (0 to 6$)$ & 0.6 ( -8 to 6$)$ & $-2.9(-3$ to -1$)$ & $-2.9(-3$ to -1$)$ & $-7.1(-10$ to 0$)$ \\
\hline Nondiabetic $(n=55)$ & $2.0(-1$ to 3$)$ & $3.0(-2$ to 6$)$ & 0.3 ( -7 to 6$)$ & $-2.9(-3$ to -1$)$ & $-2.8(-3$ to -1$)$ & $-6.9(-10$ to 1$)$ \\
\hline$p$ value & 0.572 & 0.127 & 0.778 & 0.974 & 0.475 & 0.575 \\
\hline \multicolumn{7}{|l|}{ Parity } \\
\hline$\leq 4(n=45)$ & $2(-1$ to 3$)$ & $3.0(-2$ to 6$)$ & $0.3(-8$ to 6$)$ & $-2.9(-3$ to -1$)$ & $-2.8(-3$ to -1$)$ & $-7.3(-10$ to 0$)$ \\
\hline$>4(n=25)$ & $2.2(-1$ to 3$)$ & 3.5 (0 to 6$)$ & 0.5 (-7 to 6$)$ & $-3(-3$ to -2$)$ & $-2.9(-3$ to -2$)$ & $-6.1(-9.5$ to 1$)$ \\
\hline$p$ value & 0.528 & 0.370 & 0.719 & 0.434 & 0.348 & 0.115 \\
\hline
\end{tabular}


with scores $\geq 2$ (including 2 patients with a score $=3$ ). In order to evaluate the impact of the SRS treatment on the anterior and apical compartments we analyzed this group of patients. Four of these patients presented with a descending posterior segment where the anterior and apical segments were either stage 0 or 1 . A further 2 patients presented with a global POP-Q stage $0 / 1$ prolapse and 1 patient presented at the final visit with cervical elongation (stage 1 anterior and posterior segments). There was only one patient with a positive response to question 3 who had a stage 2 anterior/apical descent, which was managed successfully using a pessary. This resulted in an overall subjective success rate of $95.7 \%$. During follow-up there were no cases with any kind of mesh exposure or with chronic pelvic pain.

All patients had completed the PFDI-20 questionnaire at their last follow-up (Table 4). There was a significant decrease in the total score (61.5 points) with a 31-point reduction in the POPDI- 6 and a 22.6-point decrease in the UDI-6 scores. Both domains met the MCID threshold for success, although this criterion was not met for the Colorectal-Anal Distress Inventory (CRAD-8) questionnaire despite a decrease being observed in this domain. Owing to the intimate nature of the sexual function questions, only 26 of the patients (37.1\%) had completed the PISQ-12 questionnaire by the last follow-up visit. There was a nonsignificant increase of 1.6 points overall reported in the sexual function scores and there were no patients with new-onset dyspareunia. In order to evaluate the impact of the SRS on the posterior compartment we analyzed the posterior compartment POP-Q measurements. Table 5 shows the Bp measurement of the posterior compartment before and after surgery for patients who did not undergo a concomitant posterior colporrhaphy with their SRS implantation. At the final follow-up 24 of the patients in this group (43.6\%) had a second-degree prolapse of the posterior compartment compared with just 9 of the patients with a stage 2 pre-operative diagnosis (16\%), suggesting a $27 \%$ worsening of the posterior compartment
Table 5 Posterior wall measurement (pre- and post-operative)

\begin{tabular}{lll}
\hline Stage & Pre-operative Bp $(n=55)$ & $\begin{array}{l}\text { Post-oper- } \\
\text { ative Bp } \\
(n=55)\end{array}$ \\
\hline 0 & $24(44 \%)$ & $22(40 \%)$ \\
1 & $20(36 \%)$ & $9(16 \%)$ \\
2 & $9(16 \%)$ & $24(43.6 \%)$ \\
3 & $(4 \%)$ & - \\
\hline
\end{tabular}

15 patients underwent native tissue repair posterior colporrhaphy, leaving 55 patients for analysis

prolapse following SRS placement. There was no worsening of rectocele in patients with pre-operative stage 0 or 1 rectocele. Four out of these 24 patients with a second-degree prolapse were symptomatic, 3 with $\mathrm{Bp}=0$, and 1 patient with $\mathrm{Bp}=-1 \mathrm{~cm}$. One of these patients with a symptomatic rectocele underwent a posterior colporrhaphy 6 months after the SRS implantation. Three patients who had $\mathrm{Bp}=1 \mathrm{~cm}$ were asymptomatic. There were no patients with stage 3 or 4 posterior compartment prolapse.

\section{Discussion}

This report presents a new surgical technique for the treatment of advanced ( $>90 \%$ of patients with third- and fourthdegree POP) anterior vaginal prolapse with or without apical prolapse. According to the International Urogynecological Association/International Continence Society joint report on the terminology for reporting outcomes of surgical procedures for POP the statistical analysis was performed based on the intention-to-treat set of data and meet the definition of a late follow-up of 3-5 years. To our knowledge, this is the first longer-term analysis of the clinical impact of the SRS implant in women surgically treated for anterior compartment prolapse. There was an overall anatomical success

Table 4 PFDI-20 and PISQ-12 scores with SRS implantation (baseline versus follow-up)

\begin{tabular}{lllllll}
\hline Questionnaire domains & Baseline $(N=70)$ & 12 months $(N=66)$ & 24 months $(N=63)$ & 36 months $(N=67)$ & Last $(N=70)$ & Difference \\
\hline POPDI-6 & 41.4 & $10.6^{*}$ & $13.8^{*}$ & $10.6^{*}$ & $10.4^{*}$ & 31.0 \\
CRAD-8 & 24.1 & $14.5^{*}$ & $17.9^{*}$ & $15.6^{*}$ & $16.1^{*}$ & 8.0 \\
UDI-6 & 40.3 & $15.4^{*}$ & $19.2^{*}$ & $17.6^{*}$ & $43.9^{*}$ & $17.7^{*}$ \\
Total PFDI-20 & 105.8 & $40.6^{*}$ & $50.9^{*}$ & $34.3^{*}$ & 61.5 \\
PISQ-12 & 31.2 & 35.4 & 33 & $32.9(\mathrm{n}=25)$ & $32.8(n=26)$ & 1.6 \\
& $(n=43)$ & $(n=32)$ & $(\mathrm{n}=33)$ & & & \\
\hline
\end{tabular}

Differences were calculated between the baseline score and the score at the last follow-up visit. The mean follow-up for patient-completed questionnaires was 38.4 months

POPDI-6Pelvic Organ Prolapse Distress Inventory, CRAD-8Colorectal-Anal Distress Inventory, UDI-6 Urine Incontinence Score, $P I S Q-12$ Pelvic Organ Prolapse/Urinary Incontinence Sexual Questionnaire

$* p<0.05$ 
rate of $94.3 \%(100 \%$ if the measured $\mathrm{Ba} \leq 0)$ and a $95.7 \%$ subjective improvement based upon question number 3 of the PFDI-20 quality-of-life questionnaire. In an analysis of all three PFDI-20 domains, there was a significant improvement in the symptoms of pelvic distress and urinary difficulty rather than in distressing colorectal symptomatology. These outcomes were not affected by lifestyle factors such as obesity, high parity, a history of smoking, or diabetes mellitus, which are known risk factors for pelvic prolapse [14], or by complications after mesh surgery [15].

The use of vaginal mesh for prolapse repair became established worldwide with the universal belief that the risk of recurrence is higher with NTRs than with mesh. Comparative trials are, however, heterogeneous and were often reported with short-term follow-up and different definitions of success [16]. The PROSPECT study comparing vaginal synthetic implants with NTR served as a pivotal consideration in the process of removing mesh kits from the market. All synthetic mesh implants in this study were anchored to pelvic structures and none involved ultra-light polypropylene titanized mesh. Although the study was limited to a 2-year follow-up, anatomical outcome reported for both NTR and synthetic mesh included $\mathrm{Ba}=-1.3 \mathrm{~cm}$ and $\mathrm{C}=-6 \mathrm{~cm}$. The mesh group had $7.4 \%$ mesh exposure at 12 months and 3.9\% surgical mesh removal [17]. The SRS 3-year results revealed better efficacy and safety outcomes. Despite a generally low POP recurrence rate reported with mesh, it became evident that there was a significant risk for mesh-related complications with the potential for these symptoms to negatively impact health-related quality of life and to prompt revisional surgery $[17,18]$. Our initial cadaveric work with the SRS implant showed the feasibility of recreating the anatomical structure of the pubo-cervical fascia and to provide adequate level I, II, and III support [8]. The SRS device represents a combination of chemical and mechanic characteristics that resist the buckling and wrinkling of the material thought to be implicated in mesh exposure [19]. The titanium dioxide was found to alter the pattern of shrinkage of the implanted mesh and to reduce the inflammatory reaction of the tissue. Both effects tend toward improvements in the ingrowth process, which is important in mesh-tissue integration. The mechanical effect of the solid frame allows the mesh to remain flat and in the absence of fixation to retain its position without tension [20]. This tension-free positional stability should also diminish the likelihood of collapse of the pore configuration over time, which has been shown to lead to contraction of the material under multi-axial loading [21].

Our approach is coupled with an anchorless technique where it is proposed that the complications observed with vaginal implants are largely a result of uncontrolled scar formation around points of fixation with anchored mesh kits [22]. During a 36-month follow-up in our series there was only one case of partial frame erosion and no mesh erosion, a rate of exposure far lower than the range of 3-14\% mesh erosions typically reported with anchored transvaginal mesh kits [2]. Regarding frame erosion of the SRS frame, we advocate surgical resection of the exposed part without preliminary trial of local estrogen therapy. Luo et al. suggested that in order to avoid mesh erosion over long-term follow-up, there should be a fundamental alteration of the fixation technique [23]. In this respect they have advocated an "anatomical implant technique," where they reported only a $1 \%$ rate of mesh erosion over 8 years in 175 cases. However, $10 \%$ of the patients reported chronic pain and perineal discomfort. In our study using an anchorless implant none of the patients complained of chronic pelvic or perineal pain.

Our study presents the longest SRS implant follow-up and it builds upon previously reported SRS outcome data $[9,10]$. Comparisons may be made with recently reported outcomes for other types of mesh that are still in use. In this respect, Naumann et al. [24] reported outcomes with the Calistar $S^{\circledR}$ (Promedon, Cordoba, Argentina) implant. They reported an 18-month follow-up with a composite success rate of $76 \%$, mesh erosion in $5.6 \%$, and dyspareunia in $3.7 \%$ of the cases. Using a partially absorbable mesh, Steures et al. [25] reported an $88 \%$ composite success rate over a 24-month follow-up but without any benefit compared with NTR procedures. Weintraub et al. reported a 12-month follow-up using Seratom ${ }^{\circledR}$ (Serag-Wiessner, Naila, Germany) [26] with a 5.7\% incidence of dyspareunia and recurrent prolapse in $6.8 \%$ of the patients. Allegré et al. reported a 12-month follow-up using the Uphold LITE TM (Boston Scientific, Marlborough, MA, USA) documenting an anatomical cure rate of $94 \%$ but an overall composite success rate of only $72.4 \%$ [27].

Our subjective long-term success rate was $98.6 \%$, which is comparable with other studies reporting a shorter-term follow-up [2]. Our patients had a statistically significant and clinically relevant reduction in their overall PFDI-20 scores, with the main sustainable effects of surgery on the prolapse and urinary domains. Two patients had transient post-operative voiding difficulty. This problem is common after vaginal surgery and need not necessarily be related specifically to the implant. New-onset dyspareunia was not documented in our cohort, although our patient numbers were small. The inclusion in analysis of older patients also affects the responses to these specific questions as older age groups are less likely to normally participate in regular sexual activity [28].

It is appreciated that a comparison between the SRS procedure and NTR represents a challenge as most of the literature does not present the long-term data for NTR procedures. Moreover, when referring to NTR, this reflects a heterogeneous group of surgical techniques that would each require direct comparison with the SRS implant. Concerning this point, in defining success as $\mathrm{Ba}<0$, Dias et al. [29], 
over a 2-year follow-up, favored a mesh-treated group of 33 patients over 37 women undergoing an anterior colporrhaphy ( $74.4 \%$ vs $51.2 \%$ respectively; $p=0.022$ ). In a comprehensive review of the most popular NTR procedures PazLevy et al. [30] reported $15 \%$ objective recurrence after a McCall procedure, $21 \%$ after sacrospinous ligament fixation (SSLF), a $15.3 \%$ failure rate after a utero-sacral ligament suspension (USLS), and a $21.6 \%$ incidence of de novo dyspareunia. Others reported a success rate with the USLS of $59.2 \%$ and $60.5 \%$ with the SSLF procedure. Overall, NTR as a surgical option is perceived as an acceptable operative technique, despite gradually worsening subjective and objective success. The OPTIMAL study compared SSLF with USLS and reported $13.7 \%$ anterior wall recurrence after SSLF and an $89.7 \%$ subjective success rate with the Michigan four-wall SSLF modification [30].

Of interest in our study was an apparent worsening in prolapse of the posterior compartment over time in those patients who had not undergone a concomitant posterior colporrhaphy. Our finding likely reflects the dynamic asymmetric effects of intra-abdominal pressure that may leave untreated areas vulnerable to loading forces. In these cases, the SRS might contribute to a redistribution of intra-abdominal pressure toward the posterior compartment leading to decompensation in the area opposite the implant. Withagen et al. reported a prospective observational study of 150 women who underwent a Prolift ${ }^{\mathrm{TM}}$ (Johnson and Johnson, New Brunswick, NJ, USA) repair where nearly one quarter of the patients developed a de novo POP (> stage II) in the untreated compartment. In this study there was nearly twice the rate of de novo posterior prolapse after an isolated anterior repair than anterior prolapse following an isolated posterior repair [31]. It is important to note that most of our patients with posterior wall POP currently remain asymptomatic. This observation is also in keeping with a study by Milani et al. where, in a cohort of patients with recurrent POP treated with transvaginal mesh, the presence of subsequent de novo POP in nontreated compartments did not influence the rate of surgical re-intervention [32]. Cormio et al. reported on a new NTR repair, including a large rectus fascia graft anchored in four corners to the anterior vaginal compartment. Long-term follow-up revealed 10.5\% POP recurrence and $15.8 \%$ de novo prolapse in the untreated compartment [33].

There are several limitations to our study. Although part of the follow-up visits were conducted by the surgeons, which might be considered a bias, all patients had at least one follow-up visit with a senior gynecologist other than the surgeon. The number of patients assessed is small and it is limited by its single-arm analysis. Future studies need to examine the longer-term outcomes in patients with symptomatic anterior compartment POP, comparing the SRS implant with NTRs. It is recognized that both a subjective and an objective definition of success in POP surgery is being increasingly reported in analyses. In summary, use of the SRS implant for anterior POP provides a high degree of long-term anatomical success and patient satisfaction, with minimal morbidity or risk of exposure and little need for re-intervention.

\section{Conclusion}

Our long-term findings would suggest the benefit of the SRS implant compared with other types of mesh and represents a superior outcome when compared with the long-term results of NTRs reported in the literature. Larger trials are needed in order to confirm the durable value of the SRS implant and to identify any delayed sequelae. A two-arm clinical trial is presently underway comparing the SRS implant with NTR in patients presenting with advanced stages of prolapse.

Acknowledgements We would like to thank Prof. Andrew Zbar for his support and advice in writing this manuscript.

Contributions G. Levy: project developer, manuscript writing; A. Padoa: data collector; N. Marcus: data collector; A. Beck: data collector; Z. Fekete: data collector; M. Cervigni: project development.

\section{Declarations}

Conflicts of interest G. Levy: inventor of the implant. None of the other authors has any conflicts of interest.

\section{References}

1. Siddiqi NY, Grimes CL, Casiano ER, Abed HT, Jeppson PC, Olivera CK, Society of Gynecologic Surgeons Systematic Review Group, et al. Mesh sacrocolpopexy compared with native tissue vaginal repair: a systematic review and meta-analysis. Obstet Gynecol. 2015;125:44-55. https://doi.org/10.1097/AOG.00000 000000000570.

2. Maher C, Feiner B, Baessler K, Christmann-Schmid C, Haya N, Marjoribanks J. Transvaginal mesh or grafts compared with native tissue repair for vaginal prolapse. Cochrane Database Syst Rev. 2016;2:CD012079. https://doi.org/10.1002/14651858.

3. Shah HN, Badlani GH. Mesh complications in female pelvic floor reconstructive surgery and their management: a systematic review. Ind J Urol. 2012;28:129-53. https://doi.org/10.4103/0970-1591. 98453.

4. FDA Public Health Notification: serious complications associated with transvaginal placement of surgical mesh in repair of pelvic prolapse and stress urinary incontinence. Webcache 14 February 2020 https://www.fda.gov/medical-devices/urogynecologic-surgi cal-mesh-implants/fdas-activities-urogynecologic-surgical-mesh Accessed 17 June 2021.

5. Diwadkar Gouri B, Barber Matthew D, Benjamin F, Christopher $\mathrm{M}$, Eric Jelovsek J. Complication and reoperation rates after apical vaginal prolapse surgical repair a systematic review. Obstet Gynecol. 2009;113(2 Pt 1):367-73. https://doi.org/10.1097/AOG. 0b013e318195888d. 
6. Tunn R, Rieprich M, Kaufmann O, Gauruder-Burmester A, Beyersdorff D. Morphology of the suburethral pubocervical fascia in women with stress urinary incontinence: a comparison of histologic and MRI findings. Int Urogynecol J. 2005;16:480-6. https:// doi.org/10.1007/s00192-005-1302-9.

7. Levy G, Galin A, Guy M, Marcus N, Fekete Z, Cervigni M, From A, Krissi H. Surgery for pelvic organ prolapse: the case for an anchorless implant repair. Int J Curr Med Pharmaceut Res. 2019;12A:4781-5. https://doi.org/10.24327/23955429.ijcmp r201912801.

8. Cervigni M, Ercoli A, Levy G. Cadaver study of anchorless implant for the treatment of anterior and apical vaginal wall prolapse. Eur J Obstet Gynecol Reprod Biol. 2016;23(210):173-6. https://doi.org/10.1016/j.ejogrb.2016.12.031.

9. Levy G, Padoa A, Fekete Z, Bartfai G, Pajor L, Cervigni M. Selfretaining support implant: an anchorless system for the treatment of pelvic organ prolapse-2-year follow-up. Int Urogynecol J. 2018;29:709-14. https://doi.org/10.1007/s00192-017-3415-3.

10. Levy G, Padoa A, Marcus N, Beck A, Fekete Z, Cervigni M. Anchorless implant for the treatment of advanced anterior and apical vaginal prolapse-medium term follow up. Eur J Obstet Gynecol Reprod Biol. 2020;246:55-9. https://doi.org/10.1016/j. ejogrb.2020.01.005.

11. Lowenstein L, Levy G, Chen KO, Ginath S, Condrea A, Padoa A. Validation of Hebrew versions of the Pelvic Floor Distress Inventory, Pelvic Organ Prolapse/Urinary Incontinence Sexual Function Questionnaire, and the Urgency, Severity and Impact Questionnaire. Female Pelvic Med Reconstr Surg. 2012;18:329-31. https:// doi.org/10.1097/SPV.0b013e31827268fa.

12. Barber MD, Kuchibhatla MN, Pieper CF, Bump RC. Psychometric evaluation of 2 comprehensive condition-specific quality of life instruments for women with pelvic floor disorders. Am J Obstet Gynecol. 2001;185:1388-95. https://doi.org/10.1067/mob.2001. 118659.

13. Nosti PA, Gutman RE, Iglesia CB, Park AJ, Tefera E, Sokol AI. Defining cervical elongation: a prospective observational study. J Obstet Gynaecol Can. 2017;39:223-8.

14. Vergeldt TFM, Weemhof M, IntHout J, Kluivers KB. Risk factors for pelvic organ prolapse and its recurrence: a systematic review. Int Urogynecol J. 2015;26:1559-73. https://doi.org/10. 1007/s00192-015-2695-8.

15. Deng T, Liao B, Luo D, Shen H, Wang K. Risk factors for mesh erosion after female pelvic floor reconstructive surgery: a systematic review and meta-analysis. BJU Int. 2016;117:323-43. https:// doi.org/10.1111/bju.13158.

16. Maher C, Feiner B, Baessler K, Christmann-Schmid C, Haya N, Brown J. Surgery for women with apical vaginal prolapse. Cochrane Database Syst Rev. 2016;10:CD012376. https://doi.org/ 10.1002/14651858.CD012376.

17. Glazener CM, Breeman S, Elders A, et al. Mesh, graft, or standard repair for women having primary transvaginal anterior or posterior compartment prolapse surgery: two parallel group, multicentre, randomised, controlled trials (PROSPECT). Lancet. 2017;389:381-92. https://doi.org/10.1016/S0140-6736(16) 31596-3.

18. McDonald S, Terlecki R, Costantini E, Badlani G. Complications of transvaginal mesh for pelvic organ prolapse and stress urinary incontinence: tips for prevention, recognition and management. Eur Urol Focus. 2016;2:260-7. https://doi.org/10.1016/j.euf.2016. 06.016 .

19. Feiner B, Maher C. Vaginal mesh contraction: definition, clinical presentation and management. Obstet Gynecol. 2010;115(2 Pt 1):325-30. https://doi.org/10.1097/AOG.0b013e3181cbca4d.
20. Scheidbach H, Tannapfel A, Schmidt U, Lippert H, Köckerling F. Influence of titanium coating on the biocompatibility of a heavyweight polypropylene mesh. An animal experimental model. Eur Surg Res. 2004;36:313-7. https://doi.org/10.1159/000079917.

21. Barone WR, Knight KM, Moalli PA, Abramowitch SD. Deformation of transvaginal mesh in response to multiaxial loading. J Biomech Eng. 2019;141:0210011-8. https://doi.org/10.1115/1. 4041743.

22. Margulies RU, Lewicky-Gaupp C, Fenner DE, McGuire EJ, Clemens JQ, Delancey JO. Complications requiring reoperation following vaginal mesh kit procedures for prolapse. Am J Obstet Gynecol. 2008;199:678.e1-4. https://doi.org/10.1016/j.ajog.2008. 07.049 .

23. Luo D-Y, Yang T-X, Shen H. Long term follow-up of transvaginal anatomical implant of mesh in pelvic organ prolapse. Sci Rep. 2018;8:2829. https://doi.org/10.1038/s41598-018-21090w.

24. Naumann G, Hüsch T, Mörgeli C, Kolterer A, Tunn R. Meshaugmented transvaginal repair of recurrent or complex anterior pelvic organ prolapse in accordance with the SCENIHR opinion. Int Urogynecol J. 2020;32:819-27. https://doi.org/10.1007/ s00192-020-04525-9.

25. Steures P, Milani AL, van Rumpt-van de Geest D, Kluivers KB, Withagen MIJ. Partially absorbable mesh or native tissue repair for pelvic organ prolapse: a randomized controlled trial. Int Urogynecol J. 2019;30:565-73. https://doi.org/10.1007/ s00192-108-3757-5.

26. Weintraub AY, Neuman M, Reuven Y, Neymeyer J, Marcus-Braun N. Efficacy and safety of skeletonized mesh implants for advanced pelvic organ prolapse: 12-month follow-up. World J Urol. 2016;34:1491-8. https://doi.org/10.1007/s00345-016-1792-8.

27. Allegré L, Debodinancxe P, Demattei C, Peray PF, Cayrac M, Fritel X, Courtieu C, Fatyton B, de Tayrac R. Clinical evaluation of the uphold LITE mesh for the surgical treatment of anterior and apical prolapse: a prospective, multicentre trial. Neurourol Urodyn. 2019;38:2242-9. https://doi.org/10.1002/nau.24125.

28. Bell S, Reissing ED, Henry LA, VanZuylen H. Sexual activity after 60: a systematic review of associated factors. Sex Med Rev. 2017;5:52-80. https://doi.org/10.1016/J.SXMR.2016.03.001.

29. Dias MM, Castro R de A, Bortolini MAT, Delroy CA, Martins PCF, Girão MJBC, Sartori MGF. Two-years results of native tissue versus vaginal mesh repair in the treatment of anterior prolapse according to different success criteria: a randomized controlled trial. Neurourol Urodyn. 2016;35:509-14. https://doi.org/ 10.1002/nau.22740.

30. Paz-Levy D, Yohay D, Neymeyer J, Hizkiyau R, Weintraub AY. Native tissue repair for central compartment prolapse: a narrative review. Int Urogynecol J. 2017;28:181-9. https://doi.org/10.1007/ s00192-016-3032-6.

31. Withagen MIJ, Vierhout ME, Milani AL. Does trocar-guided tension-free vaginal mesh (Prolift) repair provoke prolapse of the unaffected compartments? Int Urogynecol J. 2010;21:2718.https://doi.org/10.1007/s00192-009-1028-1.

32. Milani AL, Damoiseaux A, IntHout J, Kluivers KB, Withagen MIJ. Long-term outcome of vaginal mesh or native tissue in recurrent prolapse: a randomized controlled trial. Int Urogynecol J. 2018;29:847-58. https://doi.org/10.1007/s00192-017-3512-3.

33. Cormio L, Mancini V, Liuzzi G, d'Altilia N, Carrieri G. Surgical management of female pelvic organ prolapse with and without urinary incontinence: a single center experience. Medicine (Baltimore). 2017;96:e7914.

Publisher's note Springer Nature remains neutral with regard to jurisdictional claims in published maps and institutional affiliations. 